\title{
Acidity-dependent self-rolling of graphene oxide nanoscrolls via metal cation- $\pi$ interaction
}

\author{
Ting Shi ${ }^{1}$, Yuan $\mathrm{Yao}^{1 *}$, Yang $\mathrm{Li}^{1}$, Ningning $\mathrm{Cao}^{1}$, Jiahuang Jian ${ }^{1}$, Peng Zhang ${ }^{1}$, Songtao $\mathrm{Lu}^{1}$, Wei Qin ${ }^{2}$ and \\ Xiaohong $\mathrm{Wu}^{1^{*}}$
}

\begin{abstract}
Graphene nanoscrolls with one-dimensional topological structure obtained by Archimedean-type spirals of graphene, inherit the intrinsic properties of the pristine graphene. They have some unique advantages, including open edges/ends, adjustable internal volume, and diameter. Notably, the accommodation of functionalized components in their open interlayer is potentially a fantastic strategy to promote the epoch-making progress in nanotechnology areas, including energy storage, environmental remediation, biotechnology, and smart devices. However, it could destroy the driving forces for the self-rolling of graphene nanosheets and thus it is still a challenge to prepare functionalized graphene nanoscrolls. Here, based on density functional theory prediction, we reported a feasible method to fabricate graphene oxide nanoscrolls with carbon nanotubes as the template. The method was driven by cation- $\pi$ interactions, which were caused by metal cations that also acted as the adsorption center. Most importantly, the distinct mechanism and an acidity-dependent rule for the formation of graphene nanoscrolls were identified. Benefiting from the introduced metal cations and the macro three-dimensional hierarchical structure, the produced nanoscroll aerogel exhibited significantly improved adsorption performance toward different organic solvents with the adsorption capacities from 129.9 to $265.7 \mathrm{~g} \mathrm{~g}^{-1}$. This work demonstrates a simple and efficient strategy to fabricate functionalized component-accommodated graphene nanoscroll, which could find important applications in various fields.
\end{abstract}

Keywords: DFT prediction, cation $-\pi$ interaction, graphene oxide nanoscrolls, aerogel, adsorption capacity

\section{INTRODUCTION}

Graphene nanoscrolls, produced by rolling graphene nanosheets in a continuous manner, have drawn intensive interest as a promising type of carbon nanomaterial with open topological structures [1-4]. Besides inheriting the intrinsic characteristics of graphene, they also demonstrate some unique features, such as open edges/ends, adjustable internal volume, and diameter. Notably, the open interlayer in graphene nanoscrolls facilitates the accommodation of foreign materials for further functionalization [5-7]. Quite distinct from carbon nanotubes (CNTs) with known closed cylindrical structures, graphene nanoscrolls possess continuous $\pi$ electrons, making them excellent candidates in a wide range of applications, including energy storage and conversion [8-11], environmental remediation [12,13], as well as high-performance sensors [14-16].

Graphene is a two-dimensional (2D) planar material; thus, it is required to overcome the energy barrier in the rolling of graphene to realize the formation of graphene nanoscroll. To achieve this, certain forces (e.g., surface strain, mechanical force, and electromagnetic force) have been introduced to facilitate the rolling of graphene. Numerous strategies, including lyophilization [3], high-temperature molten salt [10], ultrasonication $[17,18]$, microexplosion [19], and refluxing [5], were therefore developed to produce graphene nanoscrolls. The nanoscrolls produced were characterized with tunable dimensions with a hierarchical carbon nanostructure consisting of graphene nanosheets and nanoscrolls. However, most current reported nanoscrolls exhibited a low-order cross-linked scroll structure, further demonstrating relatively low elasticity, strength, and thermal conductivity $[20,21]$. To overcome this, the introduction of $1 \mathrm{D}$ nanowires (e.g., vanadate oxide) as an axis had been proposed as an effective way to fabricate high-quality graphene nanoscrolls with oriented uniform structure $[22,23]$. In addition, computational studies have predicted that 1D CNTs with $\pi$ electrons could form intermolecular $\pi-\pi$ interaction with graphene, and this interaction could facilitate the rolling of graphene on the surface of CNTs [24-26]. However, experimental studies to verify the calculations were rarely reported.

Graphene oxide (GO), which is an oxidized form of graphene, could be dispersed in solution, and this allowed the liquid-based assembly of graphene [27-29]. However, due to the strong electrostatic repulsion, which arises from the surface electronegativity of GO and the charge compensation in each cycle, the rolling of GO along the surface of CNTs was found to be difficult $[30,31]$. To overcome the repulsion between GO and CNTs, the construction of covalent linkages between the aminated CNTs and the oxygen-containing group at the basal planes of GO has been shown to be a smart way to initiate the rolling of GO along the surface of the aminated CNTs [32]. However, structural distortion or collapse often occurred between adjacent slices and scrolls in the prepared GO nanoscrolls since the formation of the covalent linkages was not well controlled. Accordingly, the development of novel strategies that can

\footnotetext{
${ }^{1}$ MIIT Key Laboratory of Critical Materials Technology for New Energy Conversion and Storage, School of Chemistry and Chemical Engineering, Harbin Institute of Technology, Harbin 150001, China

${ }^{2}$ School of Materials Science and Engineering, Harbin Institute of Technology, Harbin 150001, China

* Corresponding authors (emails: wuxiaohong@hit.edu.cn (Wu X); yyuan@hit.edu.cn (Yao Y))
} 
effectively control the linkages between CNTs and GO and thus produce more desirable nanoscrolls has become a challenge.

Both GO and CNTs have continuous conjugate rings and can form strong cation- $\pi$ interactions with cations. The interactions between sodium cations and the surface of CNTs are particularly stable due to the formation of cation- $\pi$ interactions with rich $\pi$ electrons, which further mediate electron migration [33-35]. In a related cation- $\pi$ interaction system, the positively charged guanidine group of lysine in naphthalenediimide-lysine bound on the surface of CNTs, and this was shown to facilitate the selfassembly of naphthalenediimide-lysine on the surface of CNTs [36]. In addition, cation- $\pi$ interactions were utilized in metal cation-decorated GO membranes as molecular channels [37] and ion sieving [38]. Therefore, metal cations are expected to simultaneously form cation $-\pi$ interactions with GO and CNTs, and this may enable the rolling of GO on the surface of CNTs to produce nanoscrolls. Moreover, the metal cations may be able to interact with other molecules to achieve functionalization of the carbon nanoscrolls.

It is well known that metal cations with a smaller ionic radius have strong interaction with $\pi$-electrons of the benzene ring [39]. In aqueous solutions, these metal ions also possess strong shielding effects due to their high hydration energy, which weakens the cation- $\pi$ interactions with benzene rings. To predict which metal cations can simultaneously form cation- $\pi$ interactions with benzene rings in both GO and CNTs, we chose $\mathrm{Li}^{+}$, $\mathrm{Na}^{+}$, and $\mathrm{K}^{+}$cations with different ionic radius to study the interactions using density functional theory (DFT) calculations. All the three cations can be anchored on the surfaces of GO and CNTs by coordination with the oxygen-containing groups, forming stable structural units shown in Fig. 1. The frontier orbitals (see Fig. S1a-c, Supplementary information (SI)) clearly show the existence of cation- $\pi$ interactions of metal cations with $\mathrm{GO}$ and CNTs. When the metal cations were hydrated (see Fig. S1d-f), the cation- $\pi$ interactions were maintained but the distances between the cations and the $\pi$-system increased [40]. All metal cations were expected to simultaneously form cation- $\pi$ interactions with both GO and CNTs, since the $\mathrm{Li}^{+}$cation with the smallest ion radius can easily form these interactions [41].
Inspired by this, we developed a universal strategy to prepare GO-metal cations-CNTs nanoscrolls (GMCN). Based on the computational and experimental investigations, the cation- $\pi$ interactions of metal cations with both GO and CNTs that drive the rolling of GO on the surface of CNTs were determined. In addition, a detailed mechanism for the formation of GO nanoscroll was proposed. The prepared GMCN aerogels with well-aligned structure demonstrated high adsorption capacity toward various organic solvents with the maximum adsorption capacity of $265.7 \mathrm{~g} \mathrm{~g}^{-1}$. This work could inspire the design of novel carbon nanoscrolls with a uniform structure that can easily be functionalized for practical applications.

\section{EXPERIMENTAL SECTION}

\section{Materials}

Graphite powder (325 mesh, 99.8\%) was purchased from Alfa Aesar Chemical (Heysham, Lancashire, UK), and CNTs were purchased from Shenzhen Nanotech Port (Shenzhen, Guangdong, China). Inorganic salt powders such as $\mathrm{KMnO}_{4}, \mathrm{H}_{2} \mathrm{O}_{2}$, and $\mathrm{NaNO}_{3}$ and $98 \% \mathrm{H}_{2} \mathrm{SO}_{4}$ were obtained from Aladdin Industrial Corporation (Shanghai, China).

\section{Fabrications of GMCN aerogel and reduced aerogel}

GO, inorganic salt powder, and CNTs (Fig. S2) were uniformly mixed in deionized water with the certain weight ratio listed in Table S1 via ultrasonication or stirring for $30 \mathrm{~min}$. The fresh mixtures were then directly immersed into liquid nitrogen for 10 min until completely frozen. The frozen samples were placed into a lyophilizer ( $5 \mathrm{~Pa}$ for $48 \mathrm{~h}$ ). The synthesized aerogels were annealed under Ar atmosphere at $598 \mathrm{~K}$ for $2 \mathrm{~h}$ to produce the reduced aerogel. For comparison, a sample denoted as the rGOCNTs was prepared using the same procedure but without the addition of salt.

\section{Characterizations}

Microstructures of the prepared samples were analyzed by scanning electron microscopy (SEM) using Helios Nanolab600i (FEI, USA). Transmission electron microscopy (TEM) was

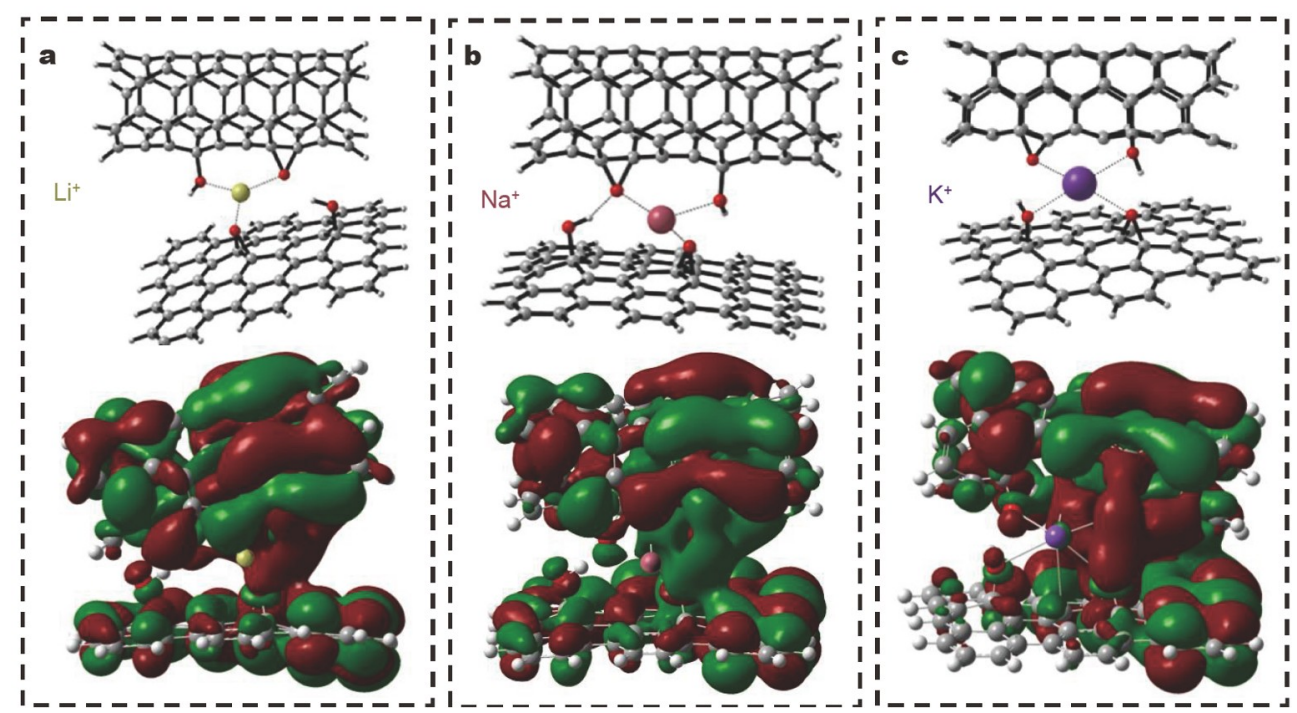

Figure 1 DFT calculations of interactions of $\mathrm{Li}^{+}, \mathrm{Na}^{+}$, and $\mathrm{K}^{+}$cations with both $\mathrm{GO}$ and CNTs. The most stable geometries and the highest occupied molecular orbitals of (a) GO- $\mathrm{Li}^{+}-\mathrm{CNTs}$, (b) GO-Na ${ }^{+}-\mathrm{CNTs}$, and (c) GO-K ${ }^{+}-\mathrm{CNTs}$. 
performed using Tecnai G2F30 (FEI, USA). For TEM, a freshly mixed solution was dropped onto a copper grid and dried for $12 \mathrm{~h}$ at room temperature. X-ray diffraction (XRD) patterns were measured using a D/max-2000 with $\mathrm{Cu}$ Ka irradiation from Rigaku corporation (Tokyo, Japan). Dynamic light scattering (DLS) and zeta potential measurements were carried out at $298 \mathrm{~K}$ using a Zetasizer Nano S (Malvern, UK). Surface hydrophilicity was measured using the sessile drop method with contact angle measurements using LSA100 from LAUDA Scientific (Württemberg, Germany). Characteristic group changes were measured by Fourier transform infrared (FT-IR) spectrometry using Nicolet iS50 from Thermo Fisher Scientific (California, USA). Specific surface areas were measured using ASAP 2460 from Micromeritics (Georgia, USA). Compression tests were carried out using Instron 5982 from Instron corporation (Massachusetts, USA). Atomic force microscopy (AFM) was performed using Dimension ICON from Bruker (Massachusetts, USA).

\section{Absorption measurement}

For absorption measurements, a reduced aerogel sample was weighed and recorded as $m_{0}$. After immersion into an organic solvent for $5 \mathrm{~min}$, the weight was measured again and recorded as $m_{1}$. The absorption capacity $(Q)$ [20] was calculated using Equation (1):

$Q=\frac{m_{1}-m_{0}}{m_{0}}$.
Each organic solvent was tested in five replicates and the average $Q$ was calculated. The recyclability of the sample was evaluated by repeating the absorption-combustion processes.

\section{Theoretical calculations}

In the DFT calculations, the geometrical structures of GMCN were optimized at B3LYP/6-31G(d) level, where the cations were located between CNTs and GO and the frequency calculations were run to identify the stationary points. The solvent effect was evaluated by adding several explicit water molecules at the same computational level. All DFT calculations were performed using Gaussian 09 software package [42].

\section{RESULTS AND DISCUSSION}

\section{The preparation and structure of GMCN aerogel}

The controlled preparation of 3D highly ordered aligned aerogel composed of 1D GMCN is illustrated in Fig. 2a. The above process consists of three sequential steps: (1) ultrasonicating or stirring the mixture of GO, CNTs, and inorganic salt (e.g., $\mathrm{Li}_{2} \mathrm{SO}_{4}$ ) to form a homogeneous dispersion for self-assembly, (2) fast freezing the fresh dispersion in a glass beaker using liquid nitrogen (Fig. S3), and (3) removal of ice crystals via freezedrying. During this process, GO nanosheets were scrolled to produce nanoscrolls (Fig. 2 and Fig. S2f). In comparison, pure $\mathrm{GO}$ in aqueous solution shows a $2 \mathrm{D}$ laminate structure (Fig. S2c). Even with the lyophilization treatment, pure GO also

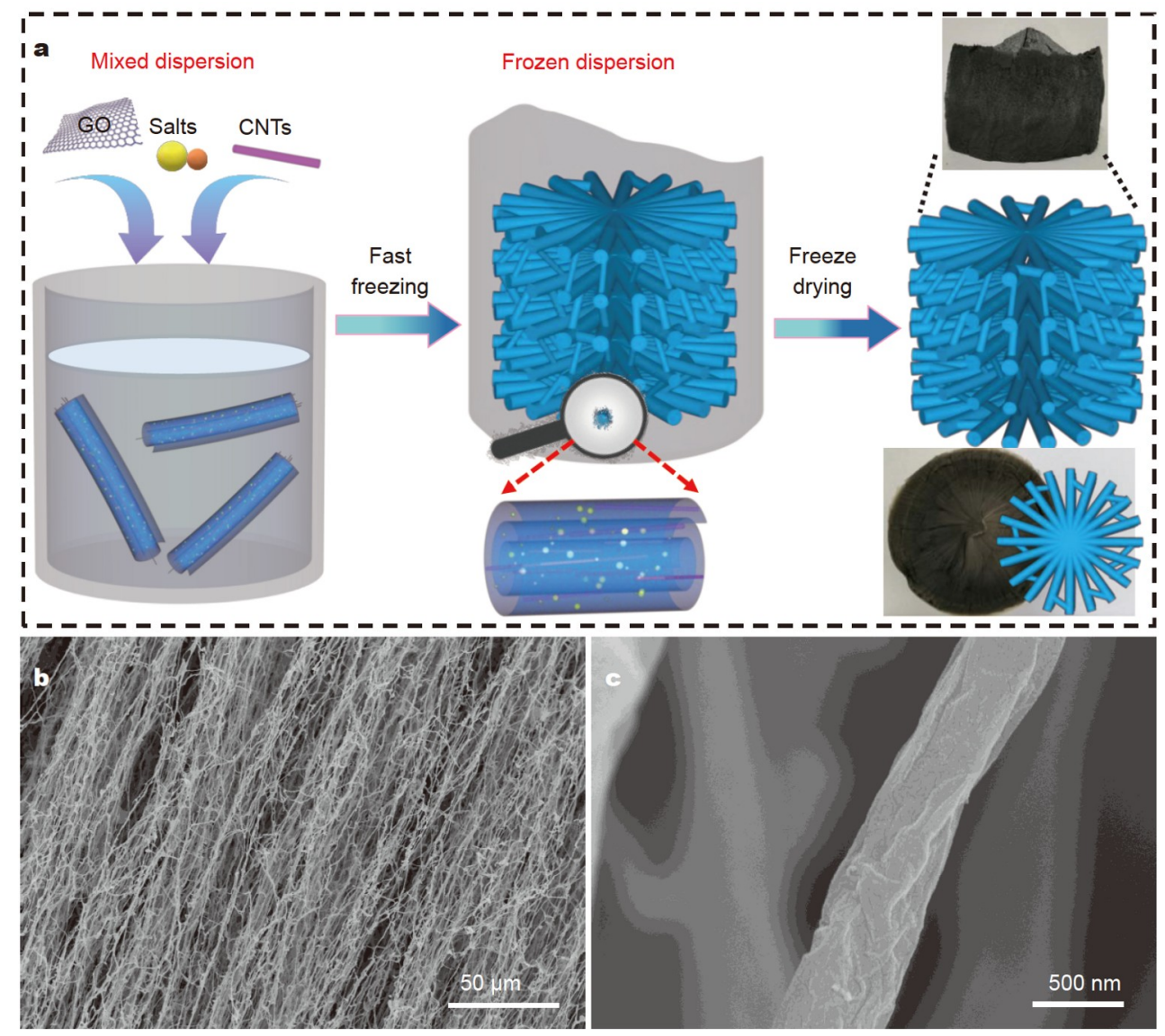

Figure 2 Structure design and morphology of the GMCN aerogel. (a) Schematic illustration of the synthesis of scroll based on GO, CNTs, and inorganic salt. $(b, c)$ SEM images of the scroll architectures. 
shows a stacking lamellar structure (Fig. S2e). As shown in Fig. 2b, c, the nanoscroll-based aerogel was uniform in size and possessed a parallelly aligned structure, rather than a disordered orientation observed in other carbon nanoscrolls [3,9,12,43]. The result in Fig. $2 \mathrm{~b}$ also shows that the produced nanoscrolls were continuously branched, thus forming a 3D network. Such a unique branched structure was also observed in a previously reported GO nanoscroll [44], constructed by adjacent GO nanosheets that were rolled and connected to each other [43]. Most notably, no single GO nanosheet was found in our sample, suggesting an almost $100 \%$ for the prepared GMCN. This yield was much higher than recently reported GO-based nanoscrolls $[3,9,12,19]$. From the AFM images (Fig. S4), the height of a nanoscroll can reach $663 \mathrm{~nm}$, and this further demonstrates the effective preparation of the nanoscroll. We note that the layer height of a GO nanosheet was only $\sim 0.8 \mathrm{~nm}$. Meanwhile, the SEM images (Fig. S5a, b) suggested that at the fracture of a nanoscroll, CNTs were wrapped in a single nanoscroll by GO nanosheets between the adjacent carbon layers. Furthermore, the TEM images (Fig. S5c, d) and energy dispersive spectrometry (EDS) mapping (Fig. S6) clearly showed that the inorganic salts were well-dispersed in the GMCN.

\section{Mechanism}

\section{Topological transformation}

There were three possible transition stages in which topological transformation of the GO can occur: (1) the fresh mixed dispersion stage, (2) the freezing stage in liquid nitrogen, and (3) the freeze-drying stage. Recent studies have speculated that the rolling was generated in the freeze-drying stage. This was due to the uneven stress experienced by the GO nanosheets inside or outside the ice. The stress was believed to be assisted by chemical reduction [3], 1-methyl-2-pyrrolidone [45], or microwave spark [2]. The formation of ice crystals during the fast cooling process in liquid nitrogen could also be a driving force for the rolling of GO [46]. Interestingly, the morphological transition of GO from nanosheet to nanoscroll can also occur in $\mathrm{N}, \mathrm{N}$-dimethylformamide (DMF) $-\mathrm{H}_{2} \mathrm{O}$ dispersions in DMF [18]. To understand the spontaneous scrolling behavior of nanoscroll, DLS [47] was employed to quantitatively detect the conformation change of the GO nanosheets. If GMCN was formed in the liquid stage, the average hydrodynamic radius distribution would significantly change [48]. As shown in Fig. 3a, the size of GO- $\mathrm{Li}_{2} \mathrm{SO}_{4}-\mathrm{CNT}$ dispersion was noticeably different from $\mathrm{GO}$ or $\mathrm{Li}_{2} \mathrm{SO}_{4}$ but very similar to CNTs. This suggested that the GO nanosheet had already rolled up to form $1 \mathrm{D}$ GMCN in aqueous solution. The two intermediate states of $1 \mathrm{D}$ GMCN in a dispersion after sonication or agitation can be identified via TEM images. As shown in Fig. 3b, c and Fig. S7, the scrolls were rolled up from the edge, while Fig. S2c presents a typical TEM image of a pure GO plane in aqueous dispersion.

The self-assembly process of 1D GMCN was found to be highly reversible in aqueous dispersion. When the prepared solutions after ultrasonication and stirring were left undisturbed for more than one hour, the scroll architecture was not achieved by fast freezing and freeze-drying. However, if the solutions were re-treated by ultrasonication and stirring, 1D GMCN (Fig. S8) formed. This was observed even when the sample was left undisturbed for more than a week. The highly reversible nature of the self-assembly process may facilitate dynamic error correction and energy minimization, generating a thermodynamically optimized composite structure with special properties [36].

\section{Key factor 1: inorganic salt featuring weak acidity}

As predicted by the DFT calculations mentioned above, metal cations could form strong cation- $\pi$ interactions with GO and CNTs to mediate the formation of $1 \mathrm{D}$ GMCN. To verify this, we performed a controlled experiment using GO-CNTs dispersion without metal cations under the same experimental condition. The results in Fig. S9 showed that GO was maintained in nanosheet conformation and not able to roll on the surface of CNTs to form a nanoscroll. This suggested that metal cations were critical for the formation of the nanoscroll. To prove the existence of cation- $\pi$ interactions between cations and aromatic ring structures in GO and CNTs, we measured the ultraviolet (UV) absorption spectra of different dispersions. As shown in Fig. 4a and Fig. S10, the maximum absorbance of GO with CNTs in water was $\sim 230 \mathrm{~nm}$, which was assigned to the $\pi-\pi^{*}$ transitions from the conjugated double bonds of an aromatic structure $[38,49,50]$. After adding metal cations, the intensity at the maximum absorbance decreased, suggesting that the conjugated double bonds were affected. This confirmed the formation of cation $-\pi$ interactions between metal cations and GO/CNTs.

The FT-IR spectrum of GO in Fig. $4 \mathrm{~b}$ showed a strong $-\mathrm{OH}$ peak at $3460 \mathrm{~cm}^{-1}, \mathrm{C}=\mathrm{O}$ bonds at $1723 \mathrm{~cm}^{-1}$, and $\mathrm{C}=\mathrm{C}$ bonds at $1630 \mathrm{~cm}^{-1}$ [51]. Meanwhile, the $\mathrm{C}=\mathrm{C}$ stretching peak of GMCN was slightly shifted, and the intensity for the absorption band of $\mathrm{C}=\mathrm{O}$ bonds decreased. This also implied the existence of the cation- $\pi$ interactions between metal cations and GO/CNTs. The cation $-\pi$ interactions between the metal cation and GO could disturb the $\mathrm{C}=\mathrm{O}$ and $\mathrm{C}=\mathrm{C}$ bonds, and this created changes in the IR signals for the $\mathrm{C}=\mathrm{C}$ stretching and stretching vibrations of
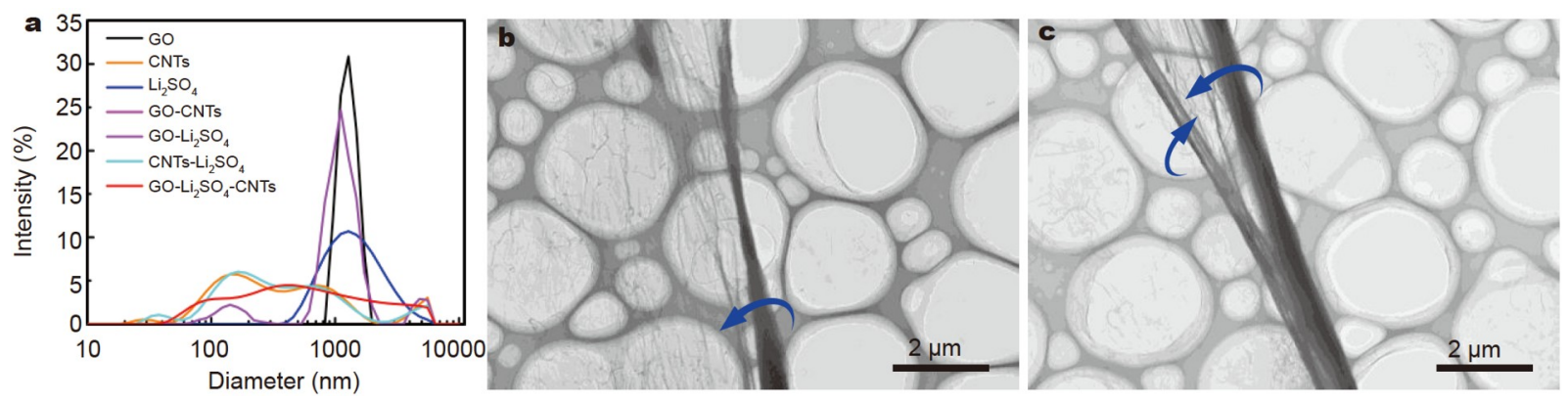

Figure 3 Topological deformation occurred on GO sheets in the process of scroll formation. (a) Size distributions of the different dispersions. (b, c) TEM images of GO-salts-CNTs scrolls in the intermediate state. 

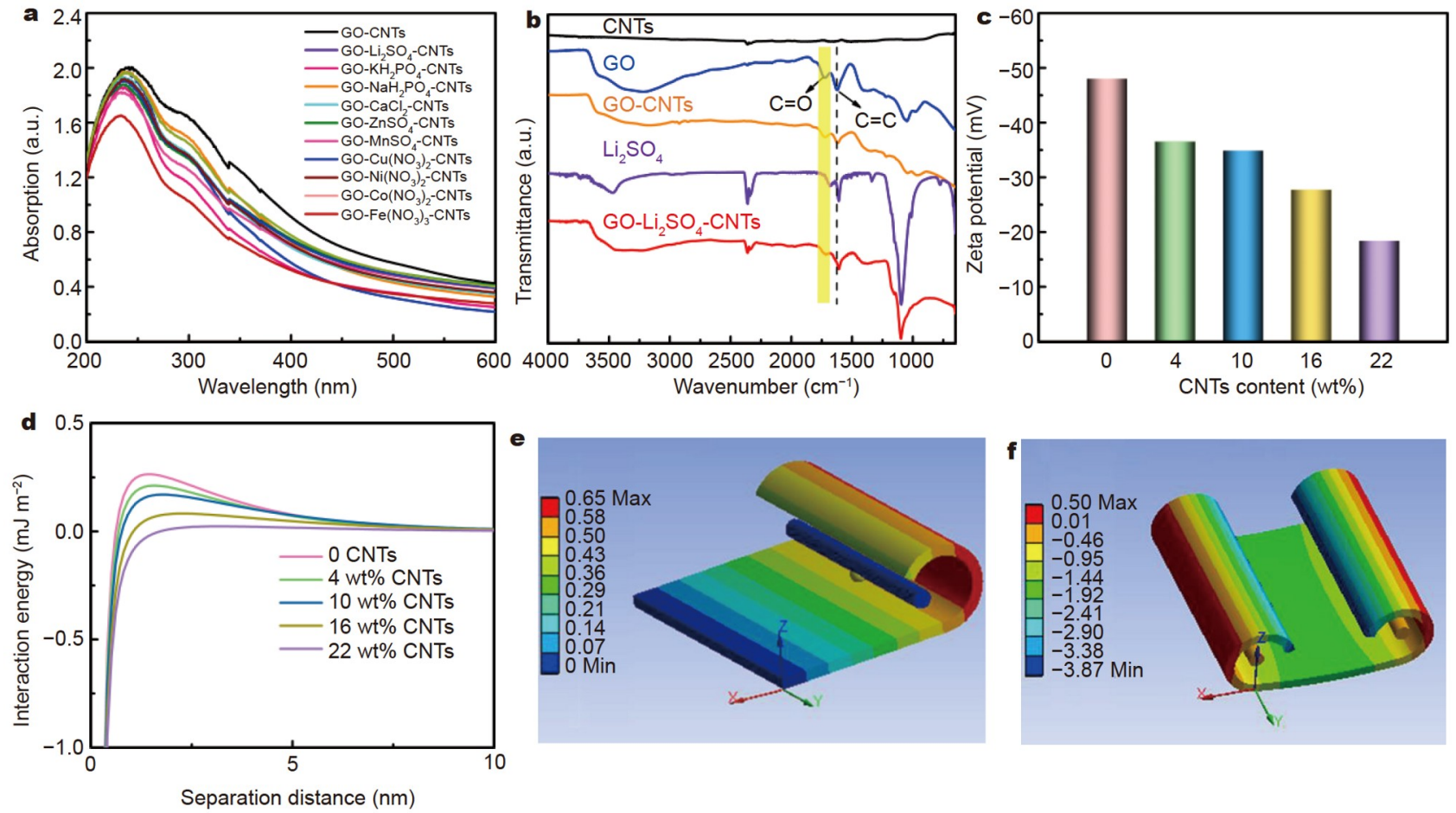

Figure 4 UV absorption spectral experiments, FT-IR, zeta measurement, DLVO theoretical calculations, and the finite element simulation for the mechanism of topological transformation. (a) UV absorption spectral experiments to demonstrate the existence of the cation- $\pi$ interactions between cations and the aromatic ring structure in GO-CNTs. (b) FT-IR of the CNTs, GO, GO-CNTs, $\mathrm{Li}_{2} \mathrm{SO}_{4}$, and $\mathrm{GO}_{-} \mathrm{Li}_{2} \mathrm{SO}_{4}-\mathrm{CNT}_{\text {T }}$ aerogel. (c) Zeta potential and (d) DLVO interaction profiles of $\mathrm{GO}-\mathrm{Li}_{2} \mathrm{SO}_{4}$-CNTs (the mass fraction of CNTs was $0,4 \%, 10 \%, 16 \%$, and $22 \%$, respectively). (e, f) Simulation modelings of the topological deformation processing.

$\mathrm{C}=\mathrm{O} \quad[38,52]$. We note that there was no evidence that GO formed covalent bonds with CNTs. Combined with the structural information, one can conclude that metal cations located between GO and CNTs can form strong cation- $\pi$ interactions in GMCN in a manner that favors the self-assembly of nanoscrolls. This supported the DFT prediction mentioned above. The impact of metal cation concentration on regulating the formation of GMCN was also investigated. The results suggested the importance of metal cation concentration (optimum salt concentration was $\sim 60 \mathrm{wt} \%$ ) in the formation of the nanoscrolls (Fig. S11, Table S2, and more details were described in SI).

To better elucidate what kind of metal cations are required to form GMCN, we set up a series of comparable experiments as listed in Table $\mathrm{S} 1$. Since $\mathrm{Li}_{2} \mathrm{SO}_{4}$ shows weak acidity $(\mathrm{pH} \sim 5.9)$, we initially studied the effect of the acid-base properties of inorganic salts on the rolling of GO. Therefore, $\mathrm{NaH}_{2} \mathrm{PO}_{4}(\mathrm{pH} \sim 5.1$ ), $\mathrm{Na}_{2} \mathrm{SO}_{4}$ ( $\mathrm{pH} \sim 6.7$ ), and $\mathrm{Na}_{3} \mathrm{PO}_{4}(\mathrm{pH} \sim 10.6)$ were used as the acidic, neutral, and alkaline salts, respectively. Fig. S12a-c suggested that the nanoscroll can only be formed with acidic salt $\mathrm{NaH}_{2} \mathrm{PO}_{4}$. Similarly, various metal cations with different valences as weakly acidic salts at $\mathrm{pH}$ between 4 and 6 , such as $\mathrm{KH}_{2} \mathrm{PO}_{4}, \quad \mathrm{CaCl}_{2}, \quad \mathrm{ZnSO}_{4}, \quad \mathrm{MnSO}_{4}, \mathrm{Cu}\left(\mathrm{NO}_{3}\right)_{2}, \mathrm{Ni}\left(\mathrm{NO}_{3}\right)_{2}$, $\mathrm{Co}\left(\mathrm{NO}_{3}\right)_{2}$, and $\mathrm{Fe}\left(\mathrm{NO}_{3}\right)_{3}$ can also mediate the formation of GMCN aerogels (Fig. S12d-k). Other acidic environments without metal ions were investigated. The addition of weak acids (acetic acid ( $\mathrm{pH} \sim 3.9$ ) or oxalic acid $(\mathrm{pH} \sim 2.9)$ ) did not allow the formation of nanoscroll (Fig. S12l, m). Even when the $\mathrm{pH}$ values were reduced to 0.1 and 2, nanoscrolls were not formed (Fig. S13). These results are consistent with the conclusion that the nanoscroll cannot be formed without metal cations discussed above. As such, both cations and a weakly acidic environment are prerequisites for the formation of the nanoscroll. For instance, when we employed neutral $\mathrm{Na}_{2} \mathrm{SO}_{4}$ as an inorganic salt and turned the $\mathrm{pH}$ of the dispersion to $<7$, the nanoscroll was formed (Fig. S12n), confirming that both the cations and weak acidic environment [53-56] are required for the formation of the nanoscroll.

\section{Key factor 2: CNTs.}

It was reported that aminated CNTs can act as a template to induce the rolling of GO on the surface of CNTs via a covalent bond between GO and CNTs [32]. As discussed above, GO and CNTs could form strong cation- $\pi$ interactions with metal cations, leading to the rolling of GO on the surface of CNTs. As such, it is important to reveal the role of CNTs in the formation of GMCN. When we performed the standard experiments without CNTs, GO nanosheets (with salt dispersed upon the surface of the GO nanosheet) still showed a planar morphology that was similar to pure GO (Fig. S14). This confirmed that CNTs play an important role in the rolling of GO. When considering GO as colloidal particles, the change of GO morphology from nanosheet to nanoscroll likely emerged from the decrease in the zeta potential $(\zeta)$ [57]. As presented in Fig. 4c, the zeta potential showed a continuous downward trend with increasing CNTs content, indicating the decrease of electronegativity. To further evaluate the effect of CNTs content on the interaction between GO and CNTs, the interaction energies (attractive van der Waals interaction $\left(\Phi_{\mathrm{vdW}}\right)$ and repulsive electrostatic doublelayer interaction $\left(\Phi_{\mathrm{EDL}}\right)$ ) were calculated according to the colloidal Derjaguin-Landau-Verwey-Overbeek (DLVO) theory (see 
Equation (2)) [58]:

$$
\Phi_{\text {Total }}=\Phi_{\mathrm{vdW}}+\Phi_{\mathrm{EDL}} \text {. }
$$

The van der Waals interaction was calculated using Equation (3):

$$
\Phi_{\mathrm{vdW}}=-\frac{A}{12 \pi h^{2}},
$$

where $A$ is the Hamaker constant $\left(6.26 \times 10^{-21} \mathrm{~J}\right)$, and $h$ is the separation distance between two particles. The electrostatic double-layer interaction was calculated using Equation (4):

$\Phi_{\mathrm{EDL}}=\varepsilon_{0} \varepsilon_{\mathrm{w}} k \psi^{2}\left[\frac{1}{\sinh (k h)}+1-\operatorname{coth}(k h)\right]$,

where $\varepsilon_{0}$ is the vacuum permittivity $\left(8.854 \times 10^{-12} \mathrm{C} \mathrm{V}^{-1} \mathrm{~m}^{-1}\right)$, and $\varepsilon_{\mathrm{w}}$ is the relative dielectric permittivity of water (78.5). The zeta potential value was used to approximate the surface potential. Debye reciprocal length $k\left(\mathrm{~nm}^{-1}\right)$ was calculated using Equation (5):

$k^{-1}=\sqrt{\frac{\varepsilon_{0} \varepsilon_{\mathrm{w}} k_{\mathrm{B}} T}{2 N_{\mathrm{A}} e^{2} I}}$,

where $k_{\mathrm{B}}$ is the Boltzmann's constant $\left(1.38064852 \times 10^{-23} \mathrm{~J} \mathrm{~K}^{-1}\right)$, $T$ is the temperature $(300 \mathrm{~K}), N_{\mathrm{A}}$ is the Avogadro number $\left(6.022140857 \times 10^{23} \mathrm{~mol}^{-1}\right), e$ is the electron charge $\left(1.6021766208 \times 10^{-19} \mathrm{C}\right)$, and $I$ is the ionic strength in $\mathrm{mol} \mathrm{L}^{-1}$. As shown in Fig. 4d, the energy barrier remarkably decreased with the increase of the CNTs content. The energy barrier without CNTs was the highest, suggesting a negligible conformation change for GO. This also means that GMCN will not form in the absence of CNTs. Standard experiments with different concentrations of CNTs (see Table S2) were performed, and the results are shown in Fig. S15. When the concentration of CNTs was lower than GO, GMCN was formed. We believe that when the concentration of CNTs was higher than GO, several CNTs interacted with one GO nanosheet. This reduced the role of CNTs as a template and blocked the rolling of GO. If this interaction was disturbed, the scroll degree of GO could be significantly affected. This was also verified by our experiments with ethanol, where ethanol prevented the rolling of GO (see Fig. S16).

To further understand the role of CNTs and metal cations on the rolling of GO, we constructed two distinguishable mechanical models, which are shown in Fig. 4e, f and Fig. S17. These employed the finite element method to model the topological change of GO nanosheets in the presence of CNTs and salts. The simulations showed that the warping of GO nanosheet was caused in one side or both sides by the constraining action of CNTs and a particle. This was consistent with the identified intermediate stages of the transformation process shown in Fig. $3 \mathrm{~b}, \mathrm{c}$ and Fig. S18. The maximum displacement values of $\sim 0.6$ and $\sim 3.9 \mu \mathrm{m}$ in the $Z$ direction were found in the rolling at one side or both sides, respectively. The edges of a GO nanosheet, which rolled up at both sides, showed a much bigger displacement than that at one side. As such, when higher concentrations of metal cations and CNTs are placed on the surface of nanosheets, the morphological transformation of GO from nanosheets to nanoscrolls will most likely occur.

\section{Driving forces}

Two primary parts in energy, including the electrostatic repulsion energy $\left(W_{\mathrm{e}}\right)$ and the van der Waals interaction energy
$\left(W_{\mathrm{vdW}}\right)$ among GO, CNTs, and metal salts, dominate the formation of GO nanoscroll from a nanosheet on the surface of CNTs [18]. This can be characterized by Equation (6):

$\Delta W=W_{\mathrm{e}}+W_{\text {vdW }}$.

Therefore, the driving forces for the rolling of GO nanosheet must be strong enough to overcome the strain of the nanosheet and the electrostatic repulsion. As discussed above, the obtained experimental results supported that the GMCN could be formed in the homogeneous mixture. Hence, the driving forces for the rolling of GO nanosheet could not be the growth of the ice crystal or the sublimation of ice.

As discussed above, metal cations can simultaneously form strong cation- $\pi$ interactions with GO and CNTs which could be the major driving force for the rolling of GO nanosheet. Uniform distribution of GO, CNTs, and cations in the solution can be achieved by ultrasonication or stirring, facilitating the cation$\pi$ interactions. Under this condition, GO and CNTs will be in closer proximity to each other and hence form stable units with metal cations $[37,38]$. Once multiple cation- $\pi$ interactions were formed between the adjacent radial positions of CNTs and the same GO nanosheet, the GO nanosheet bent and subsequently wrapped along the surface of CNTs. This initiated the spontaneous rolling of the GO nanosheet and clearly indicated the template role of CNTs for the rolling of the GO nanosheet. Moreover, the spaces between GO and CNTs due to the intercalation of cations were maintained, markedly weakening the electrostatic repulsion between the oxygen-containing functional groups at the surfaces of GO and CNTs.

The weak acidic environment was considered as the secondary driving force for forming the nanoscroll. In an acidic environment, the oxygen-containing functional groups at the surfaces of GO and CNTs tend to be more protonated, and this decreases the electronegativities of both GO and CNTs [57]. The weakened electrostatic repulsion between $\mathrm{GO}$ and CNTs favored the topological transformation in the mixed solutions.

\section{Growth of ice crystal}

In the reaction mixtures, the formed GMCN was considered unordered without any constraints. When frozen in bidirectional cold sources (see Fig. S3), the radial growth of ice crystals was not in a lamellar shape, unlike in pure water. This was due to the constraints imposed by the formed nanoscrolls, resulting in a decreased volume and irregular shape, which are accompanied by a change in both thickness and roughness [20]. The nanoscrolls were pushed parallel to the bottom of the container, hence producing a highly ordered architecture (see Fig. S19). During the sublimation of ice in the freeze-drying step, this highly ordered aerogel was adopted by the nanoscrolls to generate a $3 \mathrm{D}$ macro-ordered structure.

\section{Application}

To show the advantages of the prepared aerogel, we also tested GO- $\mathrm{Li}_{2} \mathrm{SO}_{4}$-CNTs nanoscroll aerogels. The size of this $3 \mathrm{D}$ aerogel (Fig. S20a) could be flexibly controlled by using different beakers during the preparation process. As expected, the aerogel had a lower density of $1.733 \times 10^{-3} \mathrm{~g} \mathrm{~cm}^{-3}$. The diameter and height were $3.5 \mathrm{~cm}$ and $1.5 \mathrm{~cm}$, respectively. The aerogel could be balanced on top of a flower without causing significant deformation to the flower (see Fig. S20b, c). Moreover, the aerogel was robust. It could recover to its original shape without structural deformation or collapse even after applying large- 
strain manual compression and compressive stress-strain tests under $40 \%$ and $80 \%$ strains, respectively (see Fig. S21). These results demonstrated the excellent mechanical properties of this porous aerogel.

To investigate the potential adsorption capability of the prepared aerogel, we performed Brunauer-Emmett-Teller measurements and obtained nitrogen adsorption-desorption isotherms and pore size distribution curves (see Fig. S22a, b). The specific surface area was $37.6 \mathrm{~m}^{2} \mathrm{~g}^{-1}$, and the total pore volume was as low as $0.05 \mathrm{~cm}^{3} \mathrm{~g}^{-1}$. Such a low pore volume would indeed facilitate liquid absorption [59]. The obtained highly ordered structure may provide an easier path for the droplet to penetrate into the aerogel. XRD results (see Fig. S22c) suggested that the wide peak at $11.0^{\circ}$ disappeared after thermal treatment, but the peak for $\mathrm{Li}_{2} \mathrm{SO}_{4}$ (JCPDS No. 15-0873) remained. After the thermal treatment, the water contact angle of the reduced aerogel significantly increased (sharply from $0^{\circ}$ to $100^{\circ}$ ), as shown in Fig. S23a. Notably, the microstructure of the nanoscroll can be maintained (Fig. S23b) after thermal treatment, compared with the primitive one without calcination process (Fig. 2b). The ultralow density, low pore volume, and hydrophobic nature make the reduced aerogel an ideal absorbent for organic solvents.

Several organic solvents, including chloroform, ethanol, cyclohexane, and acetone, were tested to study the absorption ability of the aerogel. The prepared reduced aerogel can completely absorb cyclohexane dyed by Unisol blue within $4 \mathrm{~s}$, as shown in Fig. 5a and Video S1. The absorption capacities were from 129.9 to $265.7 \mathrm{~g} \mathrm{~g}^{-1}$ (Fig. 5b), which were better than those reported for other carbon-based aerogels (Table S3). In comparison with rGO-CNTs aerogel without metal cations, the prepared reduced aerogel possessed a higher absorption ability. This means that both metal cations and scroll structure contributed significantly to the improved absorption performance. In addition, the metals in our material may have contributed to the notable absorption performance (Fig. 5c), because cations are known to have various interactions [37] with absorbents, such as coordination interaction and hydrogen bond interaction (more details in SI). Moreover, the absorption-combustion experiments in Fig. 5d showed that the prepared reduced aerogel indicated strong absorption stability, since the aerogel kept its original shape with $\sim 90 \%$ of the initial absorption capacity retained after 50 cycles. Based on the analysis above, such rational 3D scroll-based architecture has significant potential applications in environmental remediation.

\section{CONCLUSIONS}

The GO-metal cations-CNTs nanoscrolls were successfully prepared with CNTs as a template via cation- $\pi$ interactions according to the DFT prediction. By a well-controlled strategy,
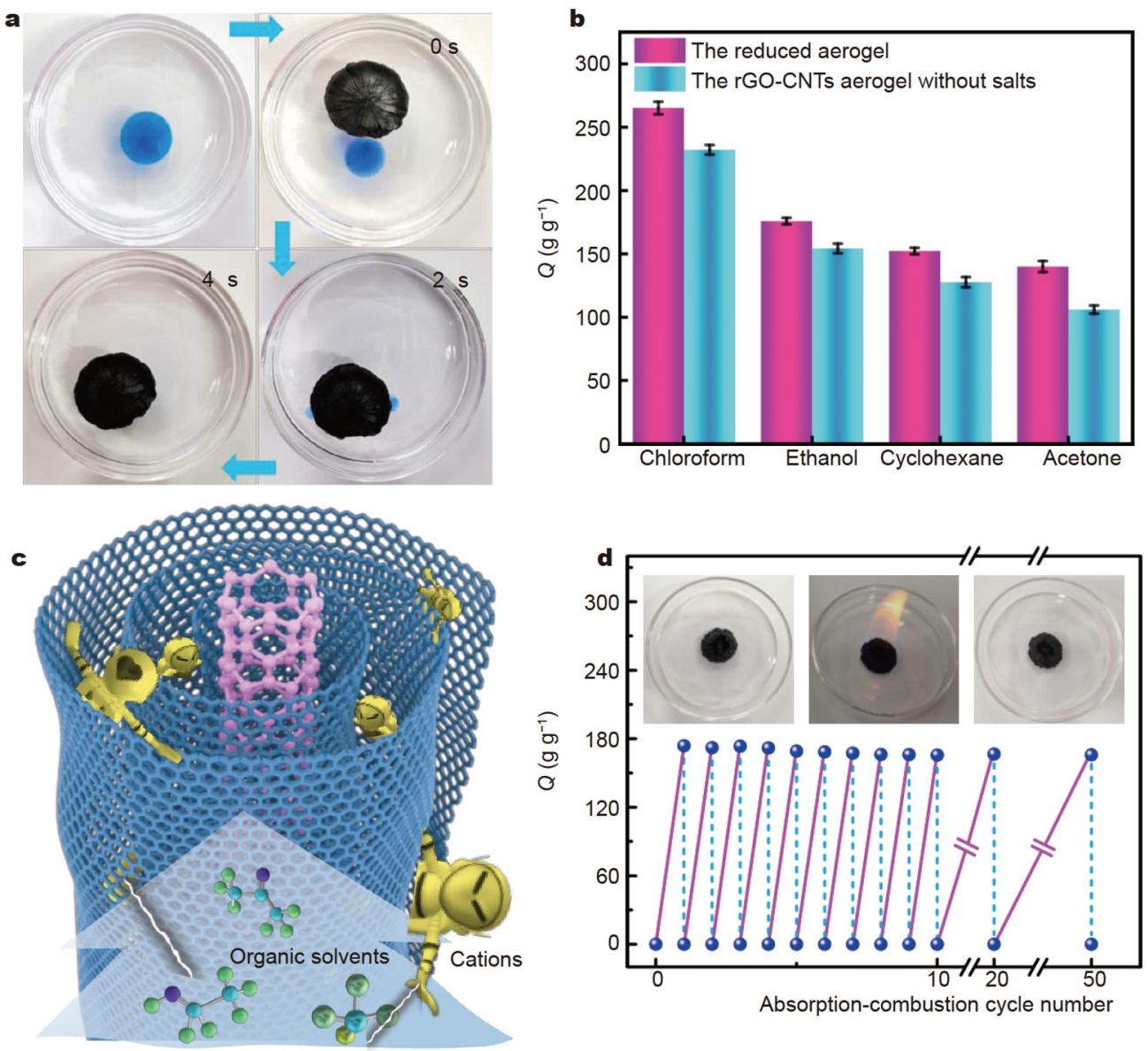

Figure 5 Application for pollution absorption. (a) Photos illustrating the fast absorption of cyclohexane dyed with Unisol blue. (b) Absorption capacities of the reduced aerogel and the control rGO-CNTs aerogel without salts. (c) Absorption mechanism model. (d) Cyclic stability of the reduced aerogel for ethanol absorption via combustion. 
the prepared nanoscrolls approached almost $100 \%$ yield, and the generated aerogel featured a highly ordered and aligned architecture. CNTs were wrapped in a single scroll by GO nanosheets between the adjacent carbon layers and the introduced inorganic salts, which were well-dispersed in the nanoscrolls. The formed cation- $\pi$ interactions were confirmed by UV and FT-IR spectrophotometry. Using DLS and other structure characterization techniques, the topological transformation stage was verified to occur in the dispersion, where the transformations were found to be reversible. Both cations and weak acidic environment were prerequisites for the formation of the nanoscrolls. On the basis of DLVO theory and finite element simulations, the role of CNTs for the rolling of GO nanosheets was also found to enhance van der Waals interactions and reduce the repulsion caused by the electrostatic double-layer via the decrease in the zeta potential. Most notably, the produced aerogel exhibited better absorption performance to various organic solvents. The absorption capacities were significantly between 129.9 and $265.7 \mathrm{~g} \mathrm{~g}^{-1}$, and thus the material could find practical applications in environmental remediation.

Received 9 September 2021; accepted 1 December 2021; published online 14 January 2022

1 Viculis LM, Mack JJ, Kaner RB. A chemical route to carbon nanoscrolls. Science, 2003, 299: 1361

2 Zheng J, Liu $\mathrm{H}, \mathrm{Wu} \mathrm{B}$, et al. Production of high-quality carbon nanoscrolls with microwave spark assistance in liquid nitrogen. Adv Mater, 2011, 23: 2460-2463

3 Xu Z, Zheng B, Chen J, et al. Highly efficient synthesis of neat graphene nanoscrolls from graphene oxide by well-controlled lyophilization. Chem Mater, 2014, 26: 6811-6818

4 Luo S, Samad YA, Chan V, et al. Cellular graphene: Fabrication, mechanical properties, and strain-sensing applications. Matter, 2019, 1: 1148-1202

5 Sharifi T, Gracia-Espino E, Reza Barzegar $\mathrm{H}$, et al. Formation of nitrogen-doped graphene nanoscrolls by adsorption of magnetic $\gamma-\mathrm{Fe}_{2} \mathrm{O}_{3}$ nanoparticles. Nat Commun, 2013, 4: 2319

6 Yang B, Chen J, Liu B, et al. One dimensional graphene nanoscrollwrapped $\mathrm{MnO}$ nanoparticles for high-performance lithium ion hybrid capacitors. J Mater Chem A, 2021, 9: 6352-6360

7 Zhang Y, Chen P, Gao X, et al. Nitrogen-doped graphene ribbon assembled core-sheath MnO@graphene scrolls as hierarchically ordered 3D porous electrodes for fast and durable lithium storage. Adv Funct Mater, 2016, 26: 7754-7765

8 Zhang Z, Zhao J, Zhou J, et al. Interfacial engineering of metal oxide/ graphene nanoscrolls with remarkable performance for lithium ion batteries. Energy Storage Mater, 2017, 8: 35-41

9 Zhao Y, Wang J, Ma C, et al. A self-adhesive graphene nanoscroll/ nanosheet paper with confined $\mathrm{Fe}_{1-x} \mathrm{~S} / \mathrm{Fe}_{3} \mathrm{O}_{4}$ hetero-nanoparticles for high-performance anode material of flexible li-ion batteries. Chem Eng J, 2019, 370: 536-546

10 Kim HK, Kamali AR, Roh KC, et al. Dual coexisting interconnected graphene nanostructures for high performance supercapacitor applications. Energy Environ Sci, 2016, 9: 2249-2256

11 Chen $\mathrm{P}$, Zhou $\mathrm{W}$, Xiao Z, et al. In situ anchoring MnO nanoparticles on self-supported 3D interconnected graphene scroll framework: A fast kinetics boosted ultrahigh-rate anode for Li-ion capacitor. Energy Storage Mater, 2020, 33: 298-308

12 Fang Q, Zhou X, Deng W, et al. Nitrogen-doped graphene nanoscroll foam with high diffusion rate and binding affinity for removal of organic pollutants. Small, 2017, 13: 1603779

13 Yap PL, Nine MJ, Hassan K, et al. Graphene-based sorbents for multipollutants removal in water: A review of recent progress. Adv Funct Mater, 2021, 31: 2007356

14 Chen Z, Wang J, Pan D, et al. Mimicking a dog's nose: Scrolling gra- phene nanosheets. ACS Nano, 2018, 12: 2521-2530

15 Li H, Wu J, Qi X, et al. Graphene oxide scrolls on hydrophobic substrates fabricated by molecular combing and their application in gas sensing. Small, 2013, 9: 382-386

16 Tung TT, Nine MJ, Krebsz M, et al. Recent advances in sensing applications of graphene assemblies and their composites. Adv Funct Mater, 2017, 27: 1702891

17 Amadei CA, Stein IY, Silverberg GJ, et al. Fabrication and morphology tuning of graphene oxide nanoscrolls. Nanoscale, 2016, 8: 6783-6791

18 Tang B, Gao E, Xiong Z, et al. Transition of graphene oxide from nanomembrane to nanoscroll mediated by organic solvent in dispersion. Chem Mater, 2018, 30: 5951-5960

19 Zeng F, Kuang Y, Wang Y, et al. Facile preparation of high-quality graphene scrolls from graphite oxide by a microexplosion method. Adv Mater, 2011, 23: 4929-4932

20 Wang $\mathrm{C}$, Chen $\mathrm{X}$, Wang B, et al. Freeze-casting produces a graphene oxide aerogel with a radial and centrosymmetric structure. ACS Nano, 2018, 12: 5816-5825

21 Cui Y, Wang Y, Shao Z, et al. Smart sponge for fast liquid absorption and thermal responsive self-squeezing. Adv Mater, 2020, 32: 1908249

22 Yan M, Wang F, Han C, et al. Nanowire templated semihollow bicontinuous graphene scrolls: Designed construction, mechanism, and enhanced energy storage performance. J Am Chem Soc, 2013, 135: $18176-18182$

23 Lin $\mathrm{Y}$, Zhou $\mathrm{F}$, Chen $\mathrm{M}$, et al. Building defect-rich oxide nanowires@graphene coaxial scrolls to boost high-rate capability, cycling durability and energy density for flexible Zn-ion batteries. Chem Eng J, 2020, 396: 125259

24 Patra N, Song Y, Král P. Self-assembly of graphene nanostructures on nanotubes. ACS Nano, 2011, 5: 1798-1804

25 Zhang Z, Li T. Carbon nanotube initiated formation of carbon nanoscrolls. Appl Phys Lett, 2010, 97: 081909

26 Wang Y, Zhan HF, Yang C, et al. Formation of carbon nanoscrolls from graphene nanoribbons: A molecular dynamics study. Comput Mater Sci, 2015, 96: 300-305

27 Muschi M, Lalitha A, Sene S, et al. Formation of a single-crystal aluminum-based MOF nanowire with graphene oxide nanoscrolls as structure-directing agents. Angew Chem Int Ed, 2020, 59: 10353-10358

28 Zhou T, Cheng Q. Chemical strategies for making strong graphene materials. Angew Chem Int Ed, 2021, 60: 18397-18410

29 Wang L, Yuan Z, Zhang Y, et al. Sandwich layered double hydroxides with graphene oxide for enhanced water desalination. Sci China Mater, 2022, 65: 803-810

$30 \mathrm{Hu} \mathrm{X}, \mathrm{Xu} \mathrm{W}$, Zhou L, et al. Tailoring graphene oxide-based aerogels for efficient solar steam generation under one sun. Adv Mater, 2017, 29: 1604031

31 Ahn E, Gaiji H, Kim T, et al. Graphene oxide nanosheet as a twodimensional polyelectrolyte: $\mathrm{pH}$-responsive behavior of a multilayered nanomembrane. J Membrane Sci, 2019, 585: 191-198

32 Kim YK, Min DH. Preparation of scrolled graphene oxides with multiwalled carbon nanotube templates. Curr Alzheimer Resbon, 2010, 48: 4283-4288

33 Pham TA, Mortuza SMG, Wood BC, et al. Salt solutions in carbon nanotubes: The role of cation $-\pi$ interactions. J Phys Chem C, 2016, 120: $7332-7338$

34 Zhang T, Matsuda H, Zhou H. Gel-derived cation- $\pi$ stacking films of carbon nanotube-graphene complexes as oxygen cathodes. ChemSusChem, 2014, 7: 2845-2852

35 Kim YT, Joo SH, Min H, et al. The exterior of single-walled carbon nanotubes as a millimeter-long cation-preferring nanochannel. Chem Mater, 2018, 30: 5184-5193

36 Ji M, Mason MKL, Modarelli DA, et al. Threading carbon nanotubes through a self-assembled nanotube. Chem Sci, 2019, 10: 7868-7877

37 Long Y, Wang K, Xiang G, et al. Molecule channels directed by cationdecorated graphene oxide nanosheets and their application as membrane reactors. Adv Mater, 2017, 29: 1606093

38 Chen L, Shi G, Shen J, et al. Ion sieving in graphene oxide membranes via cationic control of interlayer spacing. Nature, 2017, 550: 380-383

39 Bania KK, Guha AK, Bhattacharyya PK, et al. Effect of substituent and 
solvent on cation- $\pi$ interactions in benzene and borazine: a computational study. Dalton Trans, 2014, 43: 1769-1784

40 Reddy AS, Zipse H, Sastry GN. Cation- $\pi$ interactions of bare and coordinatively saturated metal ions: Contrasting structural and energetic characteristics. J Phys Chem B, 2007, 111: 11546-11553

41 Shi G, Liu J, Wang C, et al. Ion enrichment on the hydrophobic carbonbased surface in aqueous salt solutions due to cation- $\pi$ interactions. Sci Rep, 2013, 3: 3436

42 Frisch M, Trucks G, Schlegel H,et al. Gaussian 09 (revision d.01). 2009

43 Shin YE, Sa YJ, Park S, et al. An ice-templated, pH-tunable self-assembly route to hierarchically porous graphene nanoscroll networks. Nanoscale, 2014, 6: 9734-9741

44 Zhao J, Yang B, Yang Z, et al. Facile preparation of large-scale graphene nanoscrolls from graphene oxide sheets by cold quenching in liquid nitrogen. Curr Alzheimer Resbon, 2014, 79: 470-477

45 Huang X, Huang Z, Liu Q, et al. Organic solvent-assisted lyophilization: A universal method of preparing two-dimensional material nanoscrolls. ACS Omega, 2019, 4: 7420-7427

46 Fan T, Zeng W, Niu Q, et al. Fabrication of high-quality graphene oxide nanoscrolls and application in supercapacitor. Nanoscale Res Lett, 2015, 10: 192

47 Tuo W, Sun Y, Lu S, et al. Pillar[5]arene-containing metallacycles and host-guest interaction caused aggregation-induced emission enhancement platforms. J Am Chem Soc, 2020, 142: 16930-16934

48 Ye J, Zhang R, Yang W, et al. Pillar[5] arene-based [3] rotaxanes: Convenient construction via multicomponent reaction and $\mathrm{pH}$ responsive self-assembly in water. Chin Chem Lett, 2020, 31: 1550-1553

49 Yorita $\mathrm{H}$, Otomo K, Hiramatsu $\mathrm{H}$, et al. Evidence for the cation- $\pi$ interaction between $\mathrm{Cu}^{2+}$ and tryptophan. J Am Chem Soc, 2008, 130: 15266-15267

50 Shi G, Dang Y, Pan T, et al. Unexpectedly enhanced solubility of aromatic amino acids and peptides in an aqueous solution of divalent transition-metal cations. Phys Rev Lett, 2016, 117: 238102

51 Acik M, Lee G, Mattevi C, et al. Unusual infrared-absorption mechanism in thermally reduced graphene oxide. Nat Mater, 2010, 9: 840845

52 Yang K, Chen B, Zhu X, et al. Aggregation, adsorption, and morphological transformation of graphene oxide in aqueous solutions containing different metal cations. Environ Sci Technol, 2016, 50: 1106611075

53 Shih CJ, Lin S, Sharma R, et al. Understanding the $\mathrm{pH}$-dependent behavior of graphene oxide aqueous solutions: A comparative experimental and molecular dynamics simulation study. Langmuir, 2012, 28: 235-241

54 Whitby RLD, Gun'ko VM, Korobeinyk A, et al. Driving forces of conformational changes in single-layer graphene oxide. ACS Nano, 2012, 6: 3967-3973

55 Wu L, Liu L, Gao B, et al. Aggregation kinetics of graphene oxides in aqueous solutions: Experiments, mechanisms, and modeling. Langmuir, 2013, 29: 15174-15181

56 Zhao W, Wang L, Pei C, et al. Impact of $\mathrm{pH}$ on regulating ion encapsulation of graphene oxide nanoscroll for pressure sensing. Nanomaterials, 2019, 9: 548

57 He Y, Liu Y, Guo F, et al. Dynamic dispersion stability of graphene oxide with metal ions. Chin Chem Lett, 2020, 31: 1625-1629

58 Tang $\mathrm{H}$, Zhao Y, Yang X, et al. New insight into the aggregation of graphene oxide using molecular dynamics simulations and extended Derjaguin-Landau-Verwey-Overbeek theory. Environ Sci Technol, 2017, 51: 9674-9682

59 Wang C, Yang S, Ma Q, et al. Preparation of carbon nanotubes/graphene hybrid aerogel and its application for the adsorption of organic compounds. Curr Alzheimer Resbon, 2017, 118: 765-771

Acknowledgements This project was jointly supported by the National Natural Science Foundation of China (U2067216) and Heilongjiang Touyan Team (HITTY-20190033).

Author contributions Shi T carried out the experimental work, analyzed the results, and drafted the manuscript; Wu X and Yao Y conceived, designed, and coordinated the overall study and helped to analyze the results, and reviewed the paper. Li Y, Cao N, Jian J, Zhang P, Lu S, and Qin W discussed the results. All of the authors contributed to the data discussion.

Conflict of interest The authors declare no conflict of interest.

Supplementary information Experimental details and supporting data are available in the online version of the paper.

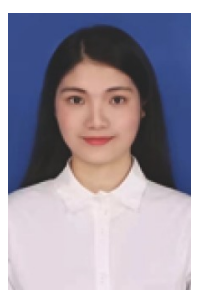

Ting Shi received her BSc degree in chemistry in 2018. Now she is a $\mathrm{PhD}$ candidate in Prof. Xiaohong Wu's group at the School of Chemistry and Chemical Engineering, Harbin Institute of Technology. Her current research is the synthesis of graphene nanoscrolls and their applications.

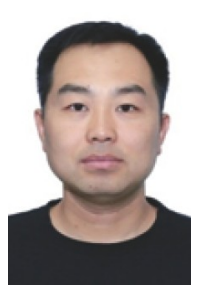

Yuan Yao received his $\mathrm{PhD}$ degree in physical chemistry from Jilin University in 2008. Currently, he is an associate professor at Harbin Institute of Technology. His research interest is the design of functional materials and their applications in energy conversion and catalysis.

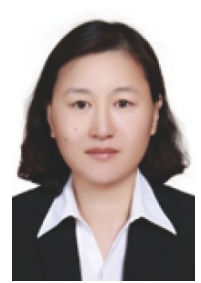

Xiaohong $\mathbf{W u}$ is a professor at Harbin Institute of Technology. She received her $\mathrm{PhD}$ degree from Harbin Institute of Technology in 2003 and was promoted to a full professor in 2010. She is the leader of the National Defense Technology Innovation Team. Her research interests include energy conversion materials, materials and devices in the extreme service environment, and surface engineering and protection.

\section{酸性环境下金属阳离子- $\pi$ 相互作用驱动氧化石墨烯 自卷曲}

石婷 ${ }^{1}$, 姚远 ${ }^{1 *}$, 李杨 ${ }^{1}$, 曹宁宁 ${ }^{1}$, 简佳煌 ${ }^{1}, 弓$ 鹏 $^{1}$, 卢松涛 ${ }^{1}$, 秦伟 ${ }^{2}$, 吴晓宏 $1^{*}$

摘要 石墨烯纳米卷作为石墨烯的一维变体结构, 它不仅继承了石墨 烯优异的本征性能, 还具有独特的开放拓扑结构. 在石墨烯纳米卷的阿 基米德型螺旋开放的层间内嵌功能组分, 可赋予石墨烯新的功能, 引领 石墨烯在能量存储、环境修复、生物技术以及智能元器件等领域实现 划时代变革. 然而, 引入功能性组分显著地削弱了石墨烯卷曲的驱动力, 因此, 制备功能化石墨烯纳米卷仍面临巨大挑战. 在本项工作中, 将密 度泛函理论预测与实验研究相结合, 我们提出了一种制备功能化石墨 烯纳米卷的普适性策略, 即以金属阳离子 $-\pi$ 相互作用为驱动力, 实现氧 化石墨烯在碳纳米管表面的自发卷曲, 获得功能化石墨烯纳米卷. 并进 一步揭示了纳米卷的形成机制和酸度依赖性规则. 基于石墨烯纳米卷 的特殊分级结构以及金属阳离子的活性吸附, 所制备的纳米卷气凝胶 表现出优异的吸附性能, 其吸附有机溶剂的容量可高达 129.9-265.7 $\mathrm{g} \mathrm{g}^{-1}$. 这项工作提供了一种简单有效且具有普适性的功能 化石墨烯纳米卷的制备策略, 可为其在相关领域的推广与应用提供理 论基础和技术支撑. 